\title{
Chiral indices of crystalline surfaces as a measure of enantioselective potential
}

\author{
Robert T. Downs ${ }^{\mathrm{a}}$, Robert M. Hazen ${ }^{\mathrm{b}, *}$

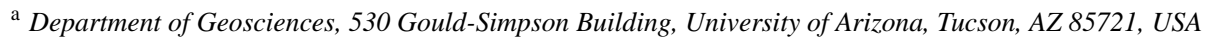 \\ ${ }^{\mathrm{b}}$ Geophysical Laboratory and NASA Astrobiology Institute, 5251 Broad Branch Road NW, Washington, DC 20015, USA
}

\begin{abstract}
Chiral crystal surfaces lack mirror or glide plane symmetry. Nevertheless, some chiral surfaces deviate more significantly from an achiral configuration, and thus possess greater enantioselective potential, than others. We describe a procedure to calculate chiral indices, $I_{\mathrm{C}}$ (in $\AA$ ), of any two-dimensional (2D) periodic atomic surface based on atomic displacements from ideal mirror or glide plane symmetry. We define a 2D unit cell parallel to the surface, identify coordinates of atoms associated with that surface unit cell, and employ minimization procedures to determine the positions and orientations of best-fit pseudo-mirror and pseudo-glide plane operators perpendicular to that surface. Achiral surfaces invariably have $I_{\mathrm{C}}=0$, but we find that surfaces of intrinsically chiral crystals [e.g., quartz $\left(\begin{array}{lll}1 & 01\end{array}\right)$ ] may also display $I_{\mathrm{C}}=0$, depending on the surface atoms selected. Of 14 surfaces modeled, $I_{\mathrm{C}}$ is greatest for chiral faces of achiral crystals: the (214) scalenohedral faces of calcite $\left(I_{\mathrm{C}}=2.60 \AA\right)$, the $\left(\begin{array}{lll}1 & 1 & 0\end{array}\right)$ faces of diopside $\left(I_{\mathrm{C}}=1.54 \AA\right)$, and the $(643)$ faces of FCC metals such as copper and platinum $\left(I_{\mathrm{C}}=1.29 \AA\right)$.
\end{abstract}

(C) 2004 Elsevier B.V. All rights reserved.

Keywords: Chiral indices; Crystalline surfaces; Enantioselective potential

\section{Introduction}

The ability of some chiral crystalline surfaces to adsorb chiral molecules and to promote heterogeneous enantioselective catalysis has received considerable recent attention from researchers in science and industry [1-9]. Prebiotic enantioselective adsorption of amino acids onto mineral surfaces has been proposed as a viable mechanism to account for the exclusive incorporation of left-handed amino acids in biological organisms [10,11]. Enantiomeric selection on crystalline surfaces, furthermore, presents a promising avenue for efficient chiral purification of pharmaceuticals and in other industrial applications [7].

In a strict crystallographic sense any periodic two-dimensional (2D) surface is either chiral or achiral, depending on whether mirrors or glide planes (both improper symmetry operators) exist perpendicular to that surface [12]. Nevertheless, some chiral arrangements of surface atoms deviate only

\footnotetext{
* Corresponding author.

E-mail address: r.hazen@gl.ciw.edu (R.M. Hazen).
}

slightly from their enantiomer, whereas other enantiomeric pairs of surfaces differ significantly from each other. In other words, some surfaces are "more chiral" than others, and thus have a greater intrinsic enantioselective potential [13]. The extent of chiral discrimination achieved for a given combination of crystal surface and chiral molecule will, of course, depend on structural details. But, lacking a detailed structural model of surface interactions, crystal surfaces with greater intrinsic enantioselectivity warrant special consideration.

No single number can characterize uniquely the "degree of chirality" of an exposed crystalline surface. Indeed, several factors, including positions of terminal atoms, their effective charge, and their bonding environments, can contribute to deviations of a crystal surface from ideal mirror or glide plane symmetry. Nevertheless, the extent to which atomic positions of a periodic 2D surface structure deviate from strict mirror or glide plane symmetry can provide the basis for a conceptually useful and mathematically well-defined "chiral index" - a measure of the enantioselective potential of that surface. This idea of a chiral index builds on a long tradition of crystal-chemical distortion indices, which have proven exceptionally useful in describing 
deviations of groups of atoms from an ideal symmetry. Thus, for example, a variety of distortion indices have been devised to characterize cation coordination polyhedra, such as $\mathrm{SiO}_{4}$ tetrahedra and $\mathrm{MgO}_{6}$ octahedra in ionic compounds [14-16]. Similarly, a distortion index described by Thompson and Downs [17] quantifies the deviation of a periodic three-dimensional oxygen array in a quasi-close-packed mineral from an ideal close-packed array. These and other distortion indices quantify the misfit of an observed atomic structure superimposed onto an idealized structure, for example, through a minimized sum of the squares of distances between observed and idealized atom positions.

This minimization strategy suggests a potentially useful approach to defining chiral indices in terms of the deviation of the observed positions of surface atoms from those of an idealized surface with mirror or glide plane symmetry. Such chiral indices define the intrinsic chirality of a surface as the extent to which that surface is non-superimposable on its enantiomer. Thus, for example, a slight distortion of an achiral surface may lead to a chiral surface with low chiral index, because the two enantiomers almost superimpose. By contrast, enantiomeric surfaces of left- and right-handed quartz $\left(\mathrm{SiO}_{4}\right)$, with opposite-handed helices of corner-linked $\mathrm{SiO}_{4}$ tetrahedra, might be predicted to have relatively large chiral indices because they are not obviously superimposable [13]. The usefulness of such a chiral index is that one can assess a priori the potential of a given surface structure for chiral selectivity. Greater misfit of enantiomeric surfaces (i.e., deviation of observed atomic coordinates from idealized mirror or glide plane symmetry) should correlate with a greater probability for an energy difference in the adsorption of molecular enantiomers, and thus a greater potential for enantioselectivity.

\section{Calculation of chiral indices}

\subsection{Computational strategy}

Two steps are necessary to calculate a chiral index. First, we specify a $2 \mathrm{D}$ periodic surface structure in terms of a surface unit cell (defined by two vectors, $\boldsymbol{a}$ and $\boldsymbol{b}$, with lengths $a$ and $b$, and an angle, $\gamma$, between them) and a set of coordinates for all atoms associated with that surface unit cell $(x, y, z)$, where $x$ and $y$ are fractional coordinates in terms of $\boldsymbol{a}$ and $\boldsymbol{b}$, and $z$ is height relative to the surface. Note that the third dimensional coordinate $z$ is required because many common crystal surfaces have atoms at varying heights.

Once a surface unit cell has been defined, then we generate a comprehensive range of fictive mirror and glide plane symmetry operators perpendicular to the surface, compute fictive atom coordinates based on those operators, and calculate deviations of observed atom positions from the fictive atom positions. We propose two complementary chiral indices based on these deviations. The "average displacement index" $\left(I_{\mathrm{CA}}\right)$ is based on the mirror or glide plane for which the average deviation of atomic positions from ideal positions (in $\AA$ ) is minimized.

Alternatively, we report a "maximum displacement index" $\left(I_{\mathrm{CM}}\right)$, which is derived by determining the largest deviation of an observed atom position (also in $\AA$ ) from its ideal position for each possible fictive mirror or glide plane. The $I_{\mathrm{CM}}$ is the smallest of all possible maximum displacements.

\subsection{Determination of the $2 D$ unit cell}

A surface (2D) unit cell is a parallelogram defined by vectors, $\boldsymbol{a}$ and $\boldsymbol{b}$, with lengths $a$ and $b$, and an angle, $\gamma$, between them. The surface unit cell can be translated by integral steps of $\boldsymbol{a}$ and $\boldsymbol{b}$ to generate the entire surface. The symmetry of the periodic surface must conform to one of the 17 plane groups, as listed in the International Tables of Crystallography.

The choice of surface atoms and their coordinates is not unique. In this study we define the 2D structure of a surface $(h k l)$ as an idealized slice of the crystal that contains all terminal atoms in the surface [13]. Note, however, that the surface of real oxide and silicate crystals feature atoms whose positions are usually relaxed from those of the 3D crystal structure [18-21]. Similarly, real surfaces of FCC metals are known to undergo considerable fluctuations in local structure due to thermal diffusion [22,23]. For this paper, however, we employ idealized atomic coordinates from the bulk crystal, as determined from 3D diffraction experiments (e.g., for calcite [24], diopside [25], orthoclase [26], quartz [27], and FCC copper with unit-cell edge $a=3.60 \AA$ ). Most surface structures have some associated depth; therefore, we define a $z$-coordinate that provides a measure of the height of the atom in angstroms for each atom. The basis vector associated with this depth is perpendicular to the surface. The slice of atoms is generated with the interactive visualization software, XtalDraw [28].

By definition, a direct space vector, $[v]_{\mathrm{D}}=\left[\begin{array}{ll}x & y z\end{array}\right]^{\mathrm{t}}$, is perpendicular to a reciprocal lattice vector, $[\boldsymbol{h}]_{\mathrm{D} *}=[h k l]^{\mathrm{t}}(h$, $k$, and $l$ are integers), only if $h x+k y+l z=0$ (nomenclature after Boisen and Gibbs [29]: bold $\boldsymbol{v}$ denotes the name of the vector, [·] denotes the triple associated with the vector $\boldsymbol{v}$ with respect to the basis indicated by the subscript $\mathrm{D}$, and the superscript $t$ designates the transpose of the triple). A surface lattice must exist in the plane parallel to a crystal face, because the equation has solutions for integer values of $x, y$, and $z$. We generate this surface lattice by finding atoms and their translational equivalents, where the translation vector is made of integers. In general, we choose the shortest vector from the set of all such translations, and define it to be one of the axes of the surface lattice. We find another reasonably short vector that defines a primitive surface lattice and that is as close as possible to being perpendicular to the first axis. The interaxial angle, $\gamma$, is found from the inner product of these lattice vectors, $\boldsymbol{a}$ and $\boldsymbol{b}$. The coordinates of the atoms in the surface are then transformed from the 3D crystallographic basis to the new 2D surface basis. 
The basis vectors of the $2 \mathrm{D}$ unit cells of 14 surfaces examined in this study are recorded in Table 1 with the coordinates of all atoms in the cell with respect to the surface basis. Several features of these 2D unit cells should be noted.

1. The dimensions of a surface unit cell may be significantly larger than those of the 3D unit cell. This situation often arises in surfaces with relatively high-Miller-indices, such as those of FCC metals, because of the oblique intersection of the surface with the 3D unit cell.

2. The number of atoms in the $2 \mathrm{D}$ asymmetric unit commonly differs from that of the 3D asymmetric unit. In the case of high-Miller-indices, the 2D number of atoms may greatly exceed that of the 3D structure. For example, a single atom forms the 3D asymmetric unit of FCC metals, but FCC surfaces that incorporate kink sites require a minimum of five atoms. By contrast, a low Miller index surface such as $(001)$ of many layer minerals may have only one atom in the $2 \mathrm{D}$ asymmetric unit, whereas 3D structures can require more than a dozen atoms.

3. A 2D lattice with $a \neq b$ and $\gamma \neq 90^{\circ}$ lacks mirror or glide plane symmetry and thus is inherently chiral; the surface structure will have plane group symmetry P1 or P2 (see also [12]). Orthogonal 2D lattices or lattices with $a=b$ may also be chiral if at least two atoms form the asymmetric unit and at least one of those atoms is in a 2D general position $(x y)$.

\subsection{Determination of chiral indices}

We computed two separate chiral indices, $I_{\mathrm{CA}}$ and $I_{\mathrm{CM}}$, for each crystal surface. The index, $I_{\mathrm{CA}}$, is a measure of the average deviation of surface atoms from a best-fit mirror or glide plane image. Alternatively, the index, $I_{\mathrm{CM}}$, is the minimum of the family of maximum deviations of individual atoms from their mirror or glide images. The chiral indices were computed using a FORTRAN code specifically developed for this purpose. The general principle involves (1) the creation of a mirror or glide plane image of a surface; (2) the association of each atom in the surface with its closest image in the mirror or glide plane image; and (3) computation of the distances separating the pairs of mirror- or glide plane-related atoms.

The values of chiral indices associated with mirror or glide plane operations depend on both the orientation and the location of the mirror or glide plane. In the special case of lattices that contain mirror or glide plane symmetry (e.g., any orthogonal lattice or lattice with $a=b$ ), an integer vector in

Table 1

Surface (2D) unit cells $(a, b$, and $\gamma)$ and atom coordinates $(x, y, z)$

\begin{tabular}{|c|c|c|c|c|c|c|c|c|}
\hline Compound & Surface $(h k l)$ & $a(\AA)$ & $b(\AA)$ & $\gamma\left({ }^{\circ}\right)$ & Atom & $x$ & $y$ & $z$ \\
\hline \multirow[t]{14}{*}{ Calcite $^{\mathrm{a}}$} & $\left(\begin{array}{lll}1 & 0 & 4\end{array}\right)$ no $\mathrm{Ca}$ & 4.9900 & 8.0959 & 90 & $\mathrm{O} 1$ & 0.1289 & 0.9876 & 0.000 \\
\hline & & & & & $\mathrm{O} 2$ & 0.8711 & 0.4876 & 0.000 \\
\hline & $\left(\begin{array}{lll}1 & 0 & 4\end{array}\right)$ with $\mathrm{Ca}$ & 4.9900 & 8.0959 & 90 & Ca1 & 0.5000 & 0.1397 & 0.000 \\
\hline & & & & & $\mathrm{Ca} 2$ & 0.5000 & 0.6397 & 0.000 \\
\hline & & & & & $\mathrm{O} 1$ & 0.2578 & 0.3897 & 0.000 \\
\hline & & & & & $\mathrm{O} 2$ & 0.7422 & 0.8897 & 0.000 \\
\hline & & & & & $\mathrm{O} 3$ & 0.1289 & 0.9876 & 0.783 \\
\hline & & & & & $\mathrm{O} 4$ & 0.8711 & 0.4876 & 0.783 \\
\hline & $\left(\begin{array}{lll}2 & 1 & 4\end{array}\right)$ & 13.2023 & 6.3753 & 107.208 & $\mathrm{Ca}$ & 0.0959 & 0.1714 & 0.000 \\
\hline & & & & & O1 & 0.5103 & 0.9431 & 0.346 \\
\hline & & & & & $\mathrm{O} 2$ & 0.7574 & 0.9461 & 0.393 \\
\hline & & & & & $\mathrm{O} 3$ & 0.9218 & 0.4679 & 0.739 \\
\hline & & & & & $\mathrm{O} 4$ & 0.9266 & 0.9708 & 0.787 \\
\hline & & & & & O5 & 0.3381 & 0.4956 & 1.180 \\
\hline \multirow[t]{11}{*}{ Diopside $^{b}$} & $\left(\begin{array}{lll}1 & 1 & 0\end{array}\right)-\mathrm{a}$ & 5.2510 & 6.5984 & 101.476 & $\mathrm{Ca}$ & 0.5847 & 0.3386 & 0.564 \\
\hline & & & & & $\mathrm{O} 1$ & 0.1734 & 0.1436 & 0.000 \\
\hline & & & & & $\mathrm{O} 2$ & 0.8551 & 0.7006 & 0.134 \\
\hline & & & & & $\mathrm{O} 3$ & 0.3468 & 0.6674 & 0.362 \\
\hline & $\left(\begin{array}{lll}1 & 1 & 0\end{array}\right)-\mathrm{c}$ & 5.2510 & 6.5984 & 101.476 & $\mathrm{Mg}$ & 0.4915 & 0.9662 & 0.410 \\
\hline & & & & & O1 & 0.3988 & 0.2575 & 0.000 \\
\hline & & & & & $\mathrm{O} 2$ & 0.5550 & 0.6701 & 0.535 \\
\hline & $\left(\begin{array}{lll}1 & 1 & 0\end{array}\right)$-e & 5.2510 & 6.5984 & 101.476 & $\mathrm{Mg}$ & 0.4915 & 0.9662 & 0.410 \\
\hline & & & & & O1 & 0.3988 & 0.2575 & 0.000 \\
\hline & & & & & $\mathrm{O} 2$ & 0.5550 & 0.6701 & 0.535 \\
\hline & & & & & $\mathrm{O} 3$ & 0.8574 & 0.0922 & 1.127 \\
\hline \multirow[t]{4}{*}{ Orthoclase } & $\left(\begin{array}{lll}1 & 1 & 0\end{array}\right)$ & 7.2099 & 7.7680 & 104.020 & $\mathrm{O} 1$ & 0.2278 & 0.2409 & 0.000 \\
\hline & & & & & $\mathrm{O} 2$ & 0.8528 & 0.2142 & 0.186 \\
\hline & & & & & $\mathrm{O} 3$ & 0.3750 & 0.8274 & 0.563 \\
\hline & & & & & $\mathrm{O} 4$ & 0.0001 & 0.8007 & 0.749 \\
\hline
\end{tabular}


Table 1 (Continued)

\begin{tabular}{|c|c|c|c|c|c|c|c|c|}
\hline Compound & Surface $(h k l)$ & $a(\AA)$ & $b(\AA)$ & $\gamma\left({ }^{\circ}\right)$ & Atom & $x$ & $y$ & $z$ \\
\hline \multirow[t]{8}{*}{ Quartz } & \multirow[t]{2}{*}{$\left(\begin{array}{lll}1 & 0 & 1\end{array}\right)$} & \multirow[t]{2}{*}{4.9137} & \multirow[t]{2}{*}{7.3045} & \multirow[t]{2}{*}{109.655} & $\mathrm{O} 1$ & 0.1071 & 0.8946 & 0.000 \\
\hline & & & & & $\mathrm{O} 2$ & 0.8749 & 0.4304 & 0.932 \\
\hline & \multirow[t]{4}{*}{$\left(\begin{array}{lll}0 & 1 & 1\end{array}\right)$} & \multirow[t]{4}{*}{4.9137} & \multirow[t]{4}{*}{7.3045} & \multirow[t]{4}{*}{109.655} & $\mathrm{O} 1$ & 0.1366 & 0.9537 & 0.000 \\
\hline & & & & & $\mathrm{O} 2$ & 0.6619 & 0.2026 & 0.099 \\
\hline & & & & & $\mathrm{O} 3$ & 0.2246 & 0.3281 & 0.359 \\
\hline & & & & & $\mathrm{O} 4$ & 0.9483 & 0.5770 & 0.458 \\
\hline & \multirow[t]{2}{*}{$\left(\begin{array}{lll}1 & 0 & 0\end{array}\right)$} & \multirow[t]{2}{*}{4.9137} & \multirow[t]{2}{*}{5.4047} & \multirow[t]{2}{*}{90} & $\mathrm{O} 1$ & 0.2797 & 0.7855 & 0.000 \\
\hline & & & & & $\mathrm{O} 2$ & 0.6597 & 0.4521 & 0.515 \\
\hline \multirow[t]{47}{*}{ Copper } & \multirow[t]{5}{*}{$\left(\begin{array}{lll}5 & 3 & 1\end{array}\right)$} & \multirow[t]{5}{*}{4.4091} & \multirow[t]{5}{*}{4.4091} & 99.594 & $\mathrm{Cu} 1$ & 0.2857 & 0.7143 & 0.000 \\
\hline & & & & & $\mathrm{Cu} 2$ & 0.7429 & 0.4571 & 0.609 \\
\hline & & & & & $\mathrm{Cu} 3$ & 0.2000 & 0.2000 & 1.217 \\
\hline & & & & & $\mathrm{Cu} 4$ & 0.6571 & 0.9429 & 1.826 \\
\hline & & & & & $\mathrm{Cu} 5$ & 0.1143 & 0.6857 & 2.434 \\
\hline & $\left(\begin{array}{lll}6 & 4 & 3\end{array}\right)$ & 8.0498 & 6.7350 & 111.012 & $\mathrm{Cu} 1$ & 0.4508 & 0.8361 & 0.000 \\
\hline & & & & & $\mathrm{Cu} 2$ & 0.5574 & 0.5246 & 0.230 \\
\hline & & & & & $\mathrm{Cu} 3$ & 0.6639 & 0.2131 & 0.461 \\
\hline & & & & & $\mathrm{Cu} 4$ & 0.7705 & 0.9016 & 0.691 \\
\hline & & & & & $\mathrm{Cu} 5$ & 0.8770 & 0.5902 & 0.922 \\
\hline & & & & & $\mathrm{Cu} 6$ & 0.9836 & 0.2787 & 1.152 \\
\hline & & & & & $\mathrm{Cu} 7$ & 0.0902 & 0.9672 & 1.383 \\
\hline & & & & & $\mathrm{Cu} 8$ & 0.1967 & 0.6557 & 1.613 \\
\hline & & & & & $\mathrm{Cu} 9$ & 0.3033 & 0.3443 & 1.844 \\
\hline & & & & & $\mathrm{Cu} 10$ & 0.4098 & 0.0328 & 2.074 \\
\hline & & & & & $\mathrm{Cu} 11$ & 0.5164 & 0.7213 & 2.305 \\
\hline & & & & & $\mathrm{Cu} 12$ & 0.6230 & 0.4098 & 2.535 \\
\hline & (8 74 ) & 8.0498 & 9.1783 & 95.032 & $\mathrm{Cu} 1$ & 0.2093 & 0.0903 & 0.000 \\
\hline & & & & & $\mathrm{Cu} 2$ & 0.2829 & 0.8294 & 0.159 \\
\hline & & & & & $\mathrm{Cu} 3$ & 0.3566 & 0.5658 & 0.317 \\
\hline & & & & & $\mathrm{Cu} 4$ & 0.4302 & 0.3024 & 0.476 \\
\hline & & & & & $\mathrm{Cu} 5$ & 0.5039 & 0.0388 & 0.634 \\
\hline & & & & & $\mathrm{Cu} 6$ & 0.5775 & 0.7752 & 0.793 \\
\hline & & & & & $\mathrm{Cu} 7$ & 0.6512 & 0.5116 & 0.951 \\
\hline & & & & & $\mathrm{Cu} 8$ & 0.7248 & 0.2480 & 1.110 \\
\hline & & & & & $\mathrm{Cu} 9$ & 0.7984 & 0.9844 & 1.268 \\
\hline & & & & & $\mathrm{Cu} 10$ & 0.8721 & 0.7210 & 1.427 \\
\hline & & & & & $\mathrm{Cu} 11$ & 0.9457 & 0.4574 & 1.585 \\
\hline & & & & & $\mathrm{Cu} 12$ & 0.0194 & 0.1938 & 1.744 \\
\hline & & & & & $\mathrm{Cu} 13$ & 0.0930 & 0.9302 & 1.902 \\
\hline & & & & & $\mathrm{Cu} 14$ & 0.1667 & 0.6666 & 2.061 \\
\hline & & & & & $\mathrm{Cu} 15$ & 0.2403 & 0.4032 & 2.219 \\
\hline & & & & & $\mathrm{Cu} 16$ & 0.3140 & 0.1396 & 2.378 \\
\hline & & & & & $\mathrm{Cu} 17$ & 0.3876 & 0.8760 & 2.536 \\
\hline & 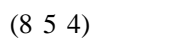 & 8.0498 & 9.1782 & 116.010 & $\mathrm{Cu} 1$ & 0.4000 & 0.0000 & 0.000 \\
\hline & & & & & $\mathrm{Cu} 2$ & 0.4810 & 0.7620 & 0.176 \\
\hline & & & & & $\mathrm{Cu} 3$ & 0.5619 & 0.5238 & 0.352 \\
\hline & & & & & $\mathrm{Cu} 4$ & 0.6429 & 0.2858 & 0.527 \\
\hline & & & & & $\mathrm{Cu} 5$ & 0.7238 & 0.0476 & 0.703 \\
\hline & & & & & $\mathrm{Cu} 6$ & 0.8048 & 0.8096 & 0.879 \\
\hline & & & & & $\mathrm{Cu} 7$ & 0.8857 & 0.5714 & 1.054 \\
\hline & & & & & $\mathrm{Cu} 8$ & 0.9667 & 0.3334 & 1.230 \\
\hline & & & & & $\mathrm{Cu} 9$ & 0.0476 & 0.0952 & 1.406 \\
\hline & & & & & $\mathrm{Cu} 10$ & 0.1286 & 0.8572 & 1.581 \\
\hline & & & & & $\mathrm{Cu} 11$ & 0.2095 & 0.6190 & 1.757 \\
\hline & & & & & $\mathrm{Cu} 12$ & 0.2905 & 0.3810 & 1.933 \\
\hline & & & & & $\mathrm{Cu} 13$ & 0.3714 & 0.1428 & 2.108 \\
\hline
\end{tabular}

a Calcite Miller indices are based on the hexagonal structural unit cell, as opposed to the morphological unit cell [13].

b Diopside $\left(\begin{array}{lll}1 & 1 & 0\end{array}\right)$ features at least three alternative surface terminations. The terminations $\left(\begin{array}{lll}1 & 1 & 0\end{array}\right)$-a, -c, and -e correspond to structures described and illustrated by Hazen [13] in Fig. 8a, c and e, respectively. 
either direct or reciprocal space can be used to describe the orientation of the symmetry plane. However, the orientation of a symmetry plane is not so straightforward to define when the lattice, itself, does not possess mirror or glide plane symmetry. On the one hand, if a mirror is defined perpendicular to an integral reciprocal lattice vector, then there is no guarantee that the separation between a given atom and its mirror image will be the same for any translation-equivalent pair because the image of lattice points may not be superimposed. Consequently, a direct space vector must be used to define the orientation of a mirror plane. In this case, we define the mirror by ${ }^{[\mathrm{uv}]} m$, where $[\boldsymbol{u} \boldsymbol{v}]$ is an integral direct space vector perpendicular to the mirror plane. The coordinates, $(u, v)$, are defined with respect to the surface bases listed in Table 1. On the other hand, a necessary condition for a glide plane is that the composition of a glide operation with itself must produce a lattice translation (i.e., defined by integers) that is perpendicular to the plane normal. This condition can be guaranteed only if the orientation of the glide plane is defined by integers in reciprocal space (again, because $h x+k y+l z=0)$. In this case, we define the glide by ${ }^{(h k)} g$, where $(h k)$ designate the integral coordinates of a reciprocal space vector that is perpendicular to the glide plane. In our algorithm, the orientations of the planes were defined by examining every mirror or glide plane with indices $h$ and $k$ between -10 and 10 [a total of 128 possible nonequivalent $(h k)]$ in the appropriate direct or reciprocal space, respectively.

We examined the effect of the location of a given symmetry plane by computing chiral indices with the plane displaced systematically from the origin of the surface unit cell. Our algorithm translated the symmetry plane across one surface unit cell, from one corner to another, displacing the plane in 100 equally spaced steps and computing the chiral indices at each step.

We computed chiral indices at each of 128 orientations and each of 100 displacements for mirror and for glide symmetry planes with the following algorithm. First, we generated the coordinates of all atoms in a $3 \times 3$ array of surface unit cells. A new Cartesian basis, designated the plane basis, was computed with its $x$-axis perpendicular to the symmetry plane, $z$-axis parallel to the surface $z$-axis, and $y$-axis perpendicular to $x$ and $z$, and consequently parallel to the plane. Note that the $y$-axis defines the direction of translation resulting from the composition of a glide with itself. The $3 \times 3$ array of surface atomic coordinates were transformed to the plane basis. We varied the origin of the plane basis for each of the 100 plane displacements, so that the plane always passed through the origin of the plane basis. Simply changing the sign of the $x$-coordinate then generates the mirror image. The glide image was computed from the mirror image by adding the translation vector determined from the composition of the glide with itself.

Each atom in the plane basis was paired with its closest atom in the mirror or glide plane image and the sep-
Table 2

Chiral indices of best-fit mirrors or glide planes computed for 14 crystal surfaces

\begin{tabular}{|c|c|c|c|c|}
\hline Compound & Surface $(h k l)$ & $I_{\mathrm{CA}}(\AA)$ & $I_{\mathrm{CM}}(\AA)$ & Orientation \\
\hline Calcite & 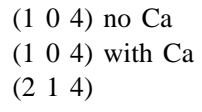 & $\begin{array}{l}0 \\
0 \\
1.73\end{array}$ & $\begin{array}{l}0 \\
0 \\
2.60\end{array}$ & $\begin{array}{l}{\left[\begin{array}{ll}0 & 1\end{array}\right]} \\
(1) \\
{\left[\begin{array}{ll}2 & 1\end{array}\right]}\end{array}$ \\
\hline Diopside & $\begin{array}{l}\left(\begin{array}{lll}1 & 1 & 0\end{array}\right)-\mathrm{a} \\
\left(\begin{array}{lll}1 & 1 & 0\end{array}\right)-\mathrm{c} \\
\left(\begin{array}{lll}1 & 1 & 0)-\mathrm{e}\end{array}\right.\end{array}$ & $\begin{array}{l}0.65 \\
0.53 \\
0.72\end{array}$ & $\begin{array}{l}1.16 \\
0.85 \\
1.54\end{array}$ & $\begin{array}{l}{\left[\begin{array}{ll}1 & 0\end{array}\right],\left[\begin{array}{ll}1 & 4\end{array}\right]} \\
{\left[\begin{array}{ll}0 & 1\end{array}\right],\left(\begin{array}{ll}1 & 0\end{array}\right)} \\
{\left[\begin{array}{ll}0 & 1\end{array}\right],\left(\begin{array}{ll}0 & 1\end{array}\right)}\end{array}$ \\
\hline Orthoclase & $\left(\begin{array}{lll}1 & 1 & 0\end{array}\right)$ & 0.52 & 1.01 & {$\left[\begin{array}{ll}1 & 0\end{array}\right]$} \\
\hline Quartz & 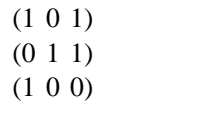 & $\begin{array}{l}0 \\
0.36 \\
0.54\end{array}$ & $\begin{array}{l}0 \\
0.46 \\
0.59\end{array}$ & $\begin{array}{l}{\left[\begin{array}{ll}1 & 0\end{array}\right]} \\
{\left[\begin{array}{ll}1 & 2\end{array}\right]} \\
{\left[\begin{array}{ll}10 & 9\end{array}\right],\left[\begin{array}{ll}1 & 0\end{array}\right]}\end{array}$ \\
\hline Copper & 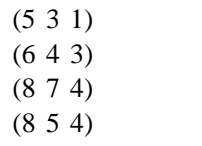 & $\begin{array}{l}0.77 \\
0.80 \\
0.85 \\
0.84\end{array}$ & $\begin{array}{l}0.96 \\
0.96 \\
1.22 \\
1.29\end{array}$ & $\begin{array}{l}{\left[\begin{array}{ll}-1 & 1\end{array}\right]} \\
{\left[\begin{array}{ll}0 & 1\end{array}\right]} \\
{\left[\begin{array}{ll}2 & 5\end{array}\right],\left[\begin{array}{ll}0 & 1\end{array}\right]} \\
{\left[\begin{array}{ll}1 & 1\end{array}\right],\left[\begin{array}{ll}0 & 1\end{array}\right]}\end{array}$ \\
\hline
\end{tabular}

The name of the mineral and its surface indices are given in the first two columns. The average displacement index, $I_{\mathrm{CA}}$, and maximum displacement index, $I_{\mathrm{CM}}$, are given in units of angstroms. The orientation of the symmetry plane is given in the last column with respect to the surface basis (see Table 1). When only one orientation is given then the symmetry planes for $I_{\mathrm{CA}}$ and $I_{\mathrm{CM}}$ coincide, and when two orientations are given, then the first is with respect to $I_{\mathrm{CA}}$. Orientations given with square brackets represent mirrors, ${ }^{[x y]} m$, and those with round brackets represent glides, ${ }^{(h k)} g$.

arations between them were determined. We computed an average index, $I_{\mathrm{A}}$, for each orientation and displacement by summing each of the atomic-pair separations, and dividing by the number of atoms; $I_{\mathrm{CA}}$ is the minimum of these indices. A maximum displacement index, $I_{\mathrm{M}}$, was computed by finding the maximum atomic-pair displacement at each orientation and displacement; $I_{\mathrm{CM}}$ is the minimum of these indices. The computed values of $I_{\mathrm{CA}}$ and $I_{\mathrm{CM}}$ along with the orientation of the best-fit mirror or glide symmetry plane for each of 14 different crystalline surfaces are given in Table 2.

An illustration of the effect of displacing a mirror across a surface unit cell is provided in Fig. 1 for the (0 1 1) surface of quartz. Fig. 1a is a plot of the average index, $I_{\mathrm{A}}$, versus the displacement of the mirror, ${ }^{[12]} m$, as it is displaced in 100 steps across the surface cell from $(00)$ to (0 1). Note that the surface unit cell has four local minima, each occurring where the pseudo mirror intersects an atom, and the global minimum occurs where the pseudo mirror is halfway between a pair of atoms and is perpendicular to the surface projection of the interatomic vector. To illustrate these pseudo mirrors, Fig. 1b displays a $3 \times 3$ array of surface unit cell contents along with fictive mirrors that pass through the local and global minima in $I_{\mathrm{A}}$. In general, local minima in the chiral index can be found when a mirror intersects an atom or when the mirror is halfway between a pair of atoms and is perpendicular to the interatomic vector. 


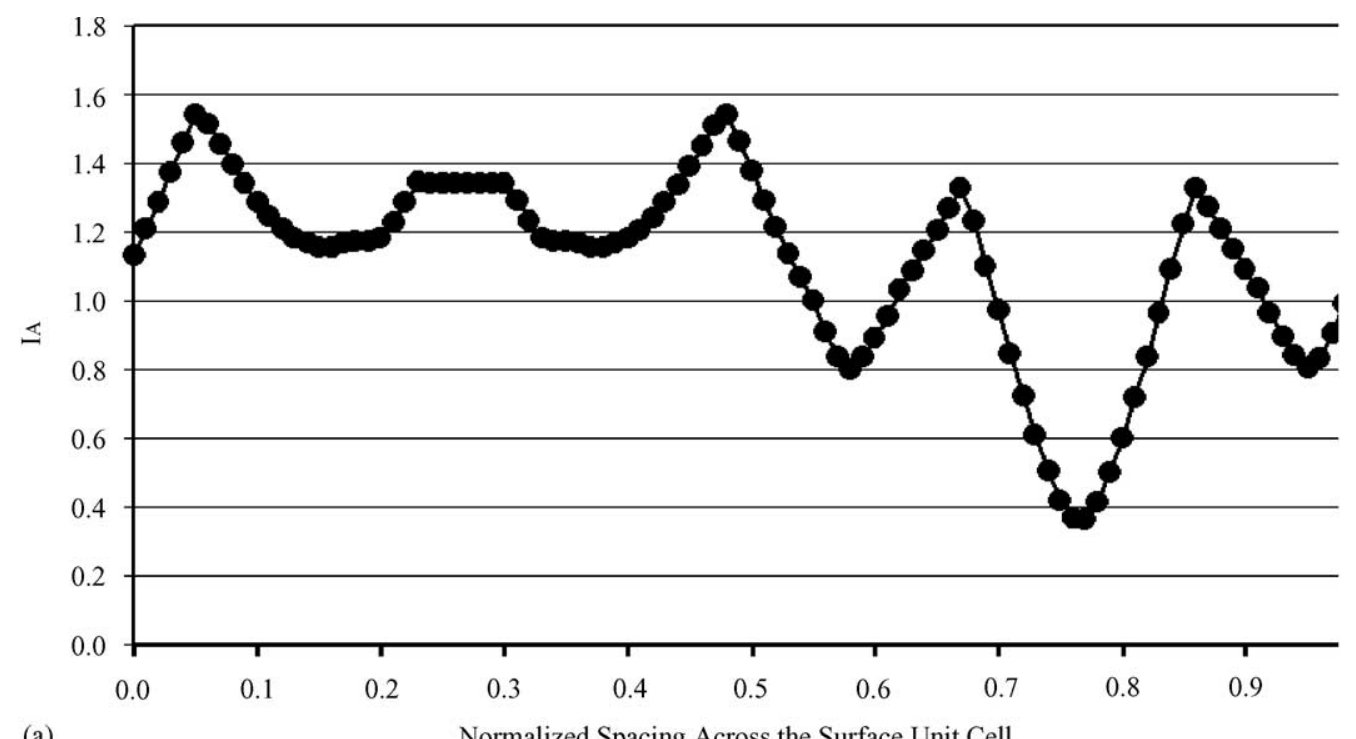

(a)

Normalized Spacing Across the Surface Unit Cell

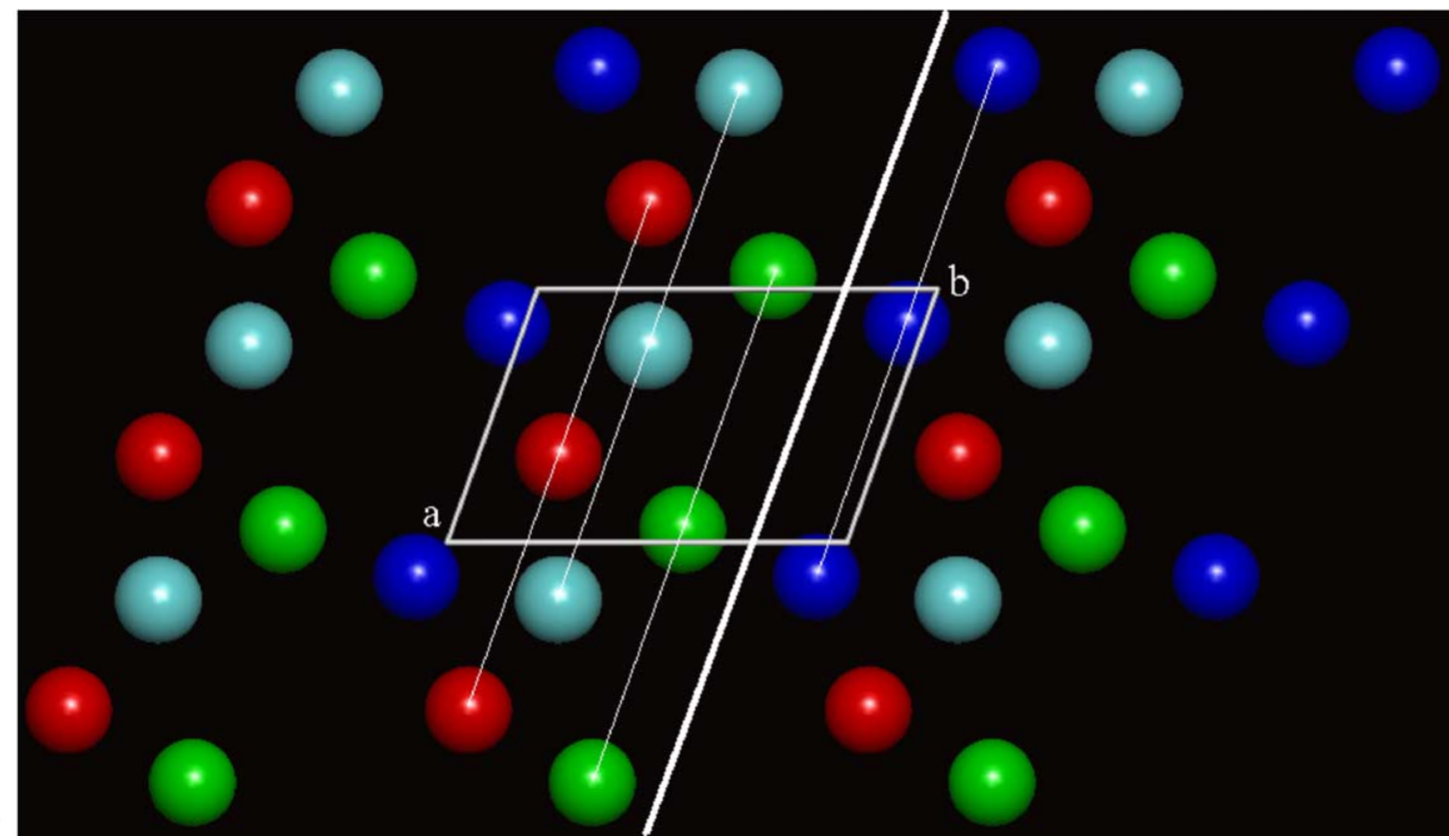

Fig. 1. Displacing a mirror across a surface unit cell has a significant effect on the chiral index, as illustrated for the ( 011$)$ surface of quartz. The pseudo mirror associated with $I_{\mathrm{CA}}$ is perpendicular to the direct space vector [12]. (a) Average chiral index, $I_{\mathrm{A}}$, plotted against the displacement of the mirror, ${ }^{[12]} \mathrm{m}$, as it is displaced in 100 steps across the surface cell from $(00)$ to $(01)$. Note that there are four local minima and one global minimum, $I_{\mathrm{CA}}$. The local minima occur for pseudo-mirrors that pass through the atomic locations. In this example, the global minimum occurs where the pseudo mirror is placed half way between pairs of atoms. (b) A $3 \times 3$ array of surface unit cell contents with a bold line that indicates the pseudo mirror associated with $I_{\mathrm{CA}}$, and four thin lines that indicate the location of the pseudo mirrors associated with the local minima in (a). The pseudo mirror associated with $I_{\mathrm{CA}}$ looks like a true mirror from this projection of the atoms; however, the mirror image pairs of atoms are at different heights.

\section{Results}

Calculated chiral indices, $I_{\mathrm{CA}}$ and $I_{\mathrm{CM}}$, for 14 different crystalline surfaces (Table 2) reveal a number of significant, and in some instances unanticipated, features. In the following sections we summarize our analyses of 10 common mineral surfaces described and illustrated by Hazen [13], including those of calcite $\left(\mathrm{CaCO}_{3}\right)$, diopside $\left(\mathrm{CaMgSi}_{2} \mathrm{O}_{6}\right)$, orthoclase $\left(\mathrm{KAlSi}_{3} \mathrm{O}_{8}\right)$, and quartz $\left(\mathrm{SiO}_{2}\right)$, as well as four different kinked surfaces of FCC copper. The face-centered cubic (FCC) unit cell edge of $\mathrm{Cu}$ is $3.60 \AA$, yielding a radius for $\mathrm{Cu}$ of $\approx 1.27 \AA$, which is similar to the radius of O. Thus, the chiral indices of $\mathrm{Cu}$ and minerals are comparably scaled.

\subsection{Calcite}

Calcite dramatically illustrates the potential for an achiral crystal to exhibit strongly chiral surfaces. The common 


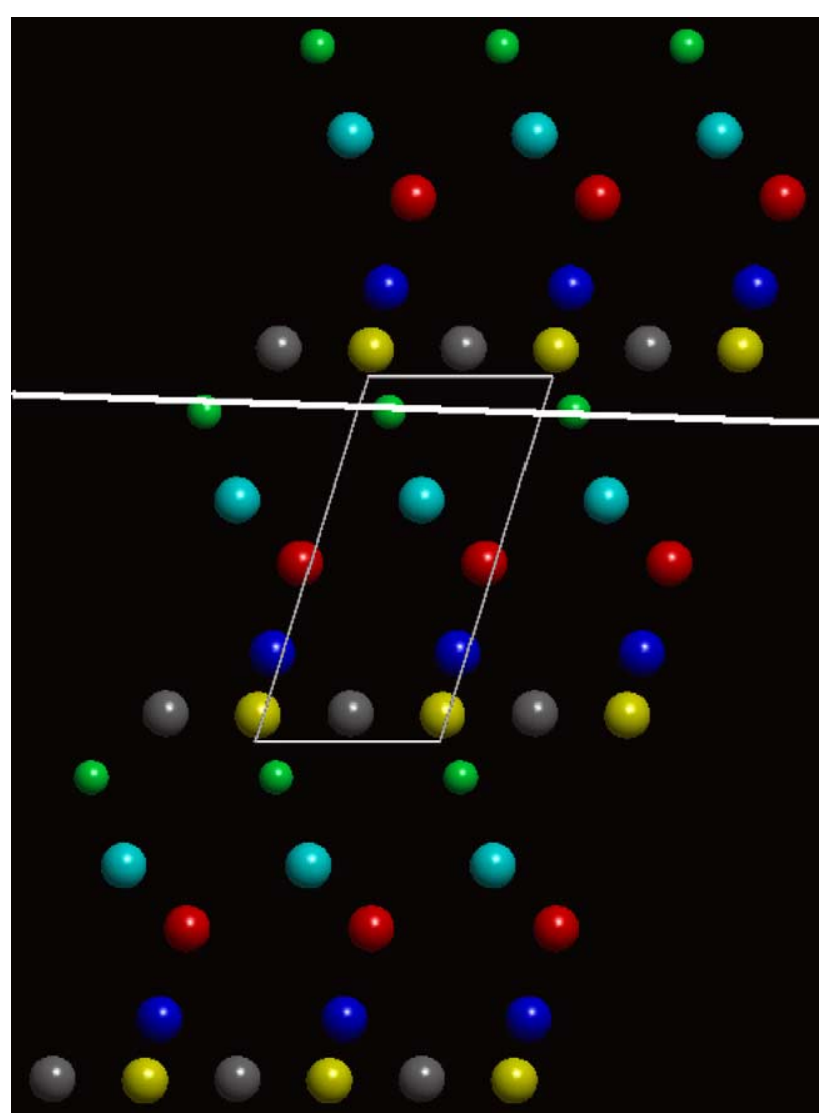

Fig. 2. Calcite $\left(\begin{array}{lll}2 & 1 & 4\end{array}\right)$ is a strongly chiral surface. The best-fit mirror (solid line) for both $I_{\mathrm{CA}}$ and $I_{\mathrm{CM}}$ is parallel to the cell edge. Smaller and larger spheres represent $\mathrm{Ca}$ and $\mathrm{O}$, respectively.

(2 14 ) scalenohedral face of calcite $\left(\mathrm{CaCO}_{3}\right)$, which is a chiral surface that adsorbs D- and L-aspartic acid differentially [11], displays by far the largest calculated chiral indices among the 14 surfaces modeled in this study. The greatest of these indices $\left(I_{\mathrm{CM}}=2.60 \AA\right)$ is comparable in magnitude to nearest neighbor anion-anion distances in many oxides and silicates and thus may represent a near-maximum possible value for $I_{\mathrm{CM}}$ of typical rock-forming minerals. This face also exhibits the largest calculated $I_{\mathrm{CA}}$ (1.73 ̊).

The reason for these relatively large chiral indices is evident from the terminal atomic structure (Fig. 2). The distribution of oxygen atoms at the calcite (214) surface shows little hint of mirror or glide plane symmetry. This atomic surface is characterized by the oblique intersection of alternating planes of $\mathrm{Ca}$ and $\mathrm{CO}_{3}$ groups - an arrangement that produces a strongly contoured, chiral surface [13]. The large chiral indices thus reflect the irregular structure, and point to the strong enantioselective potential of this surface.

By contrast, we find that the achiral calcite (1 044$)$ cleavage surface has $I_{\mathrm{CA}}=I_{\mathrm{CM}}=0 \AA$ for symmetry planes perpendicular to the [10] surface basis vector (Fig. 3), as required by symmetry constraints.

\subsection{Diopside}

Chiral (1 1 10$)$ cleavage surfaces of the common rockforming silicate diopside $\left(\mathrm{CaMgSi}_{2} \mathrm{O}_{6}\right)$ illustrate the strong dependence of chiral indices on details of surface structure. This surface can be modeled with at least three distinct arrangements of oxygen, magnesium and calcium atoms [13], depending on the presumed oxygen coordination of terminal divalent cations (Fig. 4). While details of these surface structures differ, all three feature complex distributions of $\mathrm{O}, \mathrm{Mg}$ and $\mathrm{Ca}$ that deviate from mirror or glide plane symmetry. All of these arrangements thus possess relatively large chiral indices (maximum $I_{\mathrm{CA}}=0.72 \AA$ and $I_{\mathrm{CM}}=1.54 \AA$ ), and none of these arrangements displays an obvious pseudo-mirror or pseudo-glide plane. The diopside (110) cleavage thus represents another promising surface for enantioselectivity.

\subsection{Orthoclase}

Feldspar, the commonest rock-forming mineral in Earth's crust, often features the chiral (1 10$)$ growth face. We modeled (1 110$)$ of a monoclinic potassium end-member feldspar, orthoclase $\left(\mathrm{KAlSi}_{3} \mathrm{O}_{8}\right)$. As in the case of diopside, the structural complexity of the terminal oxygen atoms, coupled with near-surface tetrahedrally coordinated $\mathrm{Al}$ and Si cations, results in a strongly chiral surface structure (Fig. 5). This face, consequently, possesses significant chiral indices $\left(I_{\mathrm{CA}}=\right.$ $0.52 \AA$ and $I_{\mathrm{CM}}=1.01 \AA$ ).

\subsection{Quartz}

Quartz $\left(\mathrm{SiO}_{2}\right)$ is the only common chiral rock-forming mineral. Its structure features helices of corner-linked $\mathrm{SiO}_{4}$ tetrahedra that can adopt either left- or right-handed configurations. One might predict, therefore, that quartz should display among the largest chiral indices. Indeed, many previous researchers employed powdered left- and right-handed quartz in studies of enantioselectivity based on this assumption [3,30,31].

Surprisingly, we find that the commonest quartz crystal growth surfaces, including (1 000$),\left(\begin{array}{llll}1 & 0 & 1\end{array}\right)$ and (1 100$)$, possess relatively small chiral indices. Indeed, the calculated chiral indices for (10 1) are zero, because the idealized terminal oxygen atom positions yield strict mirror symmetry. In this case near-surface $\mathrm{Si}$ atoms, which are not included in our idealized surface structure, will break the mirror symmetry, because surface relaxation of terminal oxygen positions will lead to shifts in surface atom positions [19]. Thus the actual (10 01 ) surface is probably somewhat chiral. Nevertheless, this quartz surface is not well suited to chiral discrimination of adsorbed molecules.

The (1 $\left.\begin{array}{lll}1 & 0\end{array}\right)$ prismatic and (lllll $\left.\begin{array}{lll}0 & 1\end{array}\right)$ rhombohedral surfaces of quartz are more promising, with maximum chiral indices of $I_{\mathrm{CA}}=0.54 \AA$ and $I_{\mathrm{CM}}=0.59 \AA$ (Table 2). Nevertheless, unlike the chiral surfaces described above for calcite, 

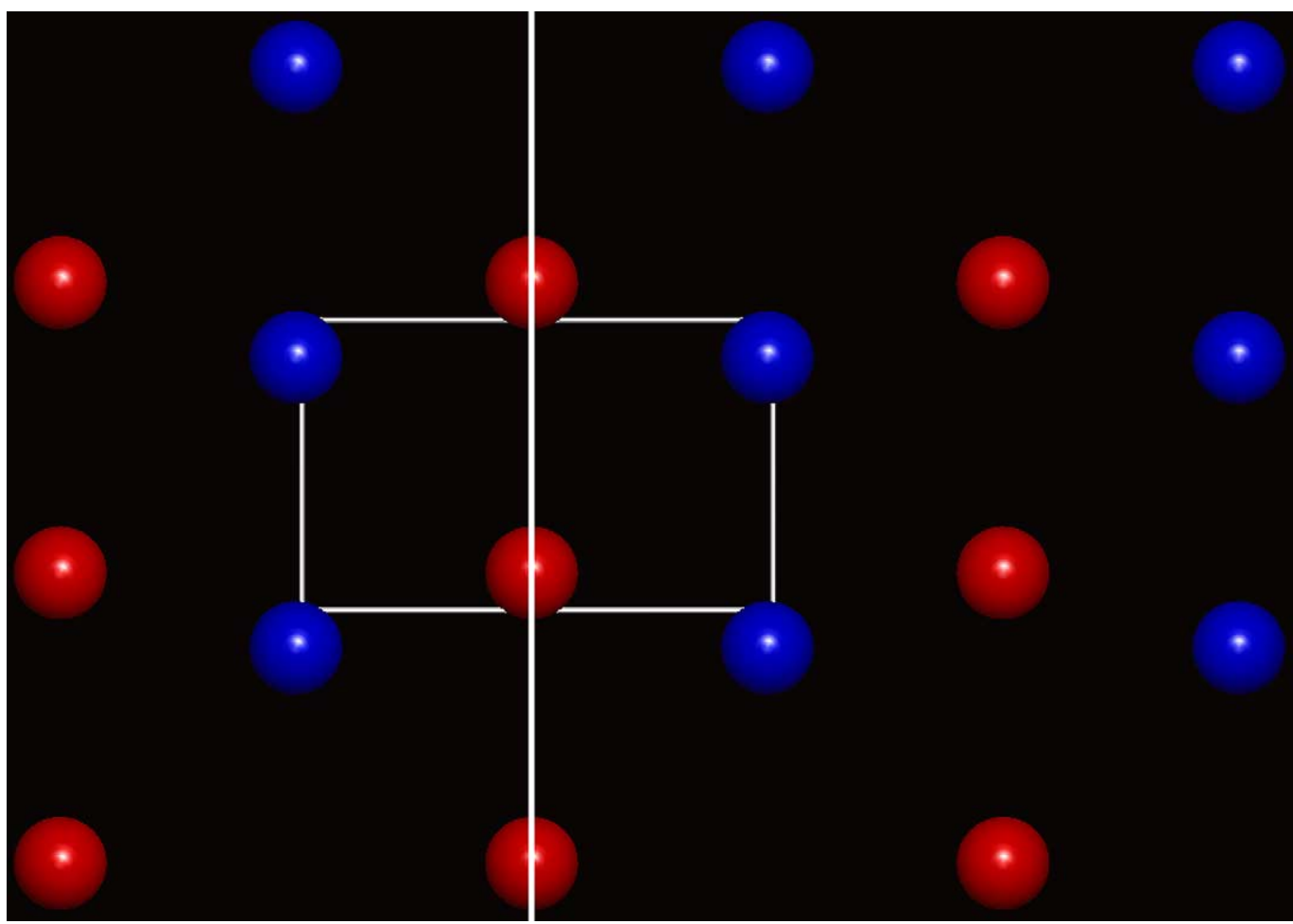

Fig. 3. The achiral $\left(\begin{array}{lll}1 & 0 & 4\end{array}\right)$ surface of calcite possesses mirror symmetry and thus has chiral indices $=0$.

diopside and orthoclase, pseudo-mirrors are clearly discernable on (100) and (011) quartz surfaces Figs. 6 and 1b, respectively).

The relatively low (or zero) chiral indices of quartz are a consequence of the constraint that every silicon atom, even those near the crystal termination, remains four-coordinated. Only oxygen atoms appear at the idealized surface, therefore, and these atoms possess a quasi-regular spacing - a topology that leads to pseudo-mirror and glide plane symmetry. The enantioselective potential of quartz thus appears, at best, to be weak compared to other common rock-forming minerals.

\subsection{Copper}

Copper, gold, platinum and silver possess the achiral face-centered cubic structure. Intuitively, it might seem that these high-symmetry metallic elements are unlikely to provide surfaces of interest in studies of chiral selection. However, a number of recent studies demonstrate that high-Miller-index planes of these metals may be cut, polished and annealed to yield chiral faces that feature periodically stepped surfaces with "kink sites" that act as chiral centers $[1,2,4,32,33]$. We modeled four of these planes-(531), (643), (874), and (854)—and find that all four possess relatively large chiral indices $\left(0.77 \leq I_{\mathrm{CA}} \leq\right.$ $0.85 \AA$ and $0.96 \leq I_{\mathrm{CM}} \leq 1.29 \AA$ ).

One unanticipated result is that the FCC (531) surface displays the lowest chiral indices among the four terminations examined, even though the (53 31 ) surface has the greatest density of kink sites (and thus a greater density of chiral centers). This result arises because our chiral indices measure not only the short-range effects of chiral centers, but also the long-range distribution of these centers about fictive mirrors or glide planes.

\section{Discussion}

\subsection{General considerations}

Data in Tables 1 and 2 lead to a number of general observations regarding chiral indices, $I_{\mathrm{CA}}$ and $I_{\mathrm{CM}}$.

1. For all mirrors and glide planes $I_{\mathrm{CA}} \leq I_{\mathrm{CM}}$, because the maximum atom displacement must be greater than or equal to the average atom displacement. In several cases, however, the best-fit symmetry planes for $I_{\mathrm{CA}}$ and $I_{\mathrm{CM}}$ differ in orientation with respect to the surface unit cell.

2. Mirrors and glide planes often yield different chiral indices. For quartz (1 00 ), diopside (1 10$)$-e, copper (8 74 ) and copper (854) mirrors provide the smallest indices, whereas for orthoclase (1 10$)$ and calcite (104) with Ca glide planes provide the minimum indices. For diopside (110)-e and copper (643) mirrors yield smaller $I_{\mathrm{CA}}$, but mirrors and glide planes are equal for $I_{\mathrm{CM}}$. For the remaining five samples, mirrors and glide planes yield identical results.

3. The orientations of best-fit mirrors and glide planes usually bear simple relationships to the surface unit cell, even though we do not constrain these orientations. For orthogonal surface unit cells, best-fit mirrors and glide 
planes are invariably parallel to one of the two unit-cell axes. For non-orthogonal surface unit cells, best-fit mirrors and glide planes are usually parallel or perpendicular to one of the unit-cell axes or to the unit-cell diagonal.

\subsection{Alternative chiral indices}

Our calculations of $I_{\mathrm{CA}}$ and $I_{\mathrm{CM}}$ for 14 surfaces demonstrate that chiral indices can provide a useful relative

(a)
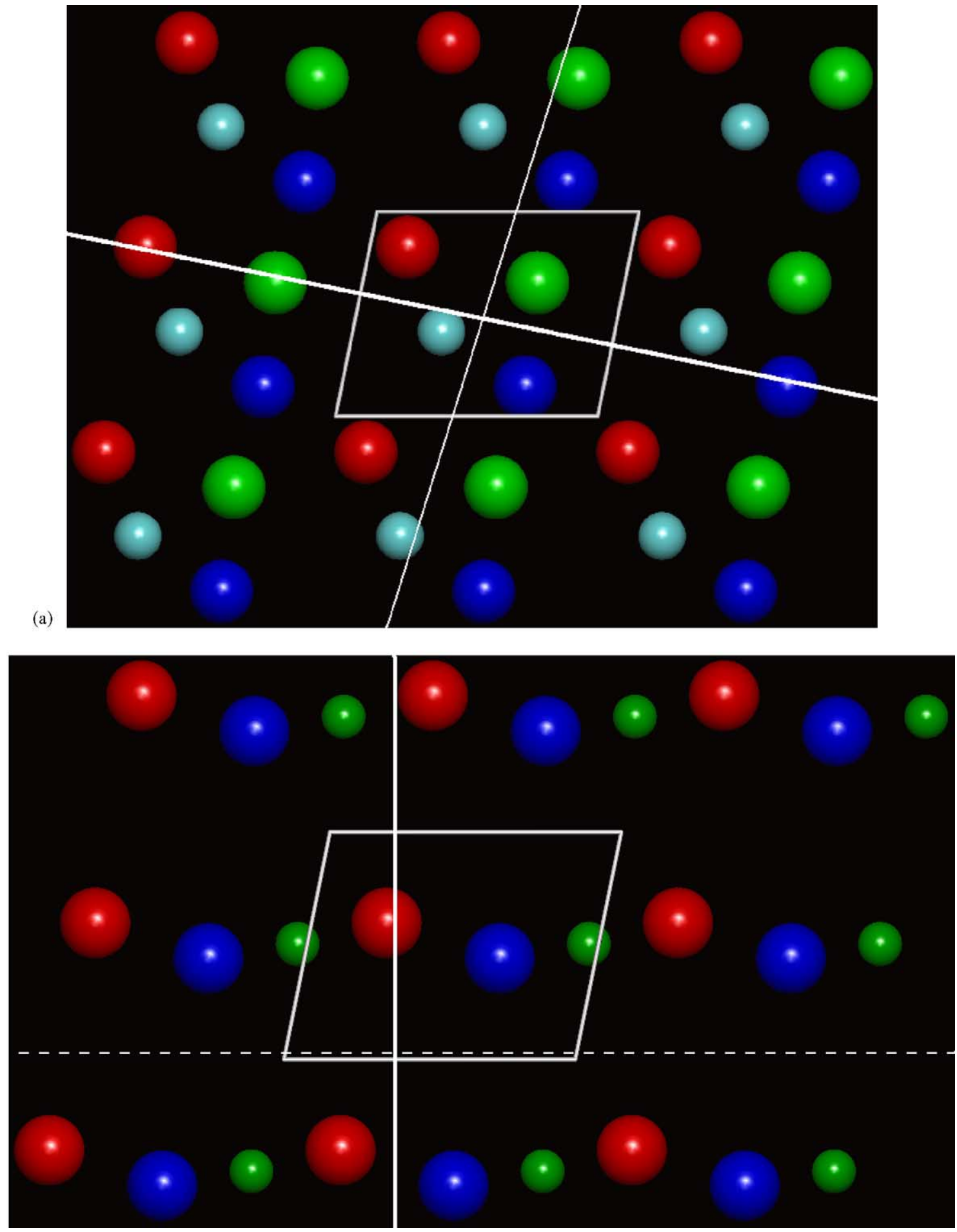

Fig. 4. Three different atomic terminations of the diopside $\left(\begin{array}{lll}1 & 1 & 0\end{array}\right)$ surface (see Table 1) result in different chiral indices and best-fit symmetry planes. Smaller and larger spheres represent cations and anions, respectively. (a) The (llll 10 )-a surface has different best-fit mirrors for $I_{\mathrm{CA}}$ (thicker line) and $I_{\mathrm{CM}}$ (thinner line), oriented parallel to [1 0 ] and [ 14 ], respectively. (b) The diopside $\left(\begin{array}{lll}1 & 1 & 0\end{array}\right)$-c surface has a best-fit mirror parallel to [0 1 l] for $I_{\mathrm{CA}}$ and a best-fit glide plane parallel to the a axis for $I_{\mathrm{CM}}$. (c) The (lllll 110 )-e surface has a best-fit mirror parallel to the b axis for $I_{\mathrm{CA}}$ (solid line), and a best-fit glide plane parallel to [0 1] for $I_{\mathrm{CM}}$ (dashed line). 
(c)

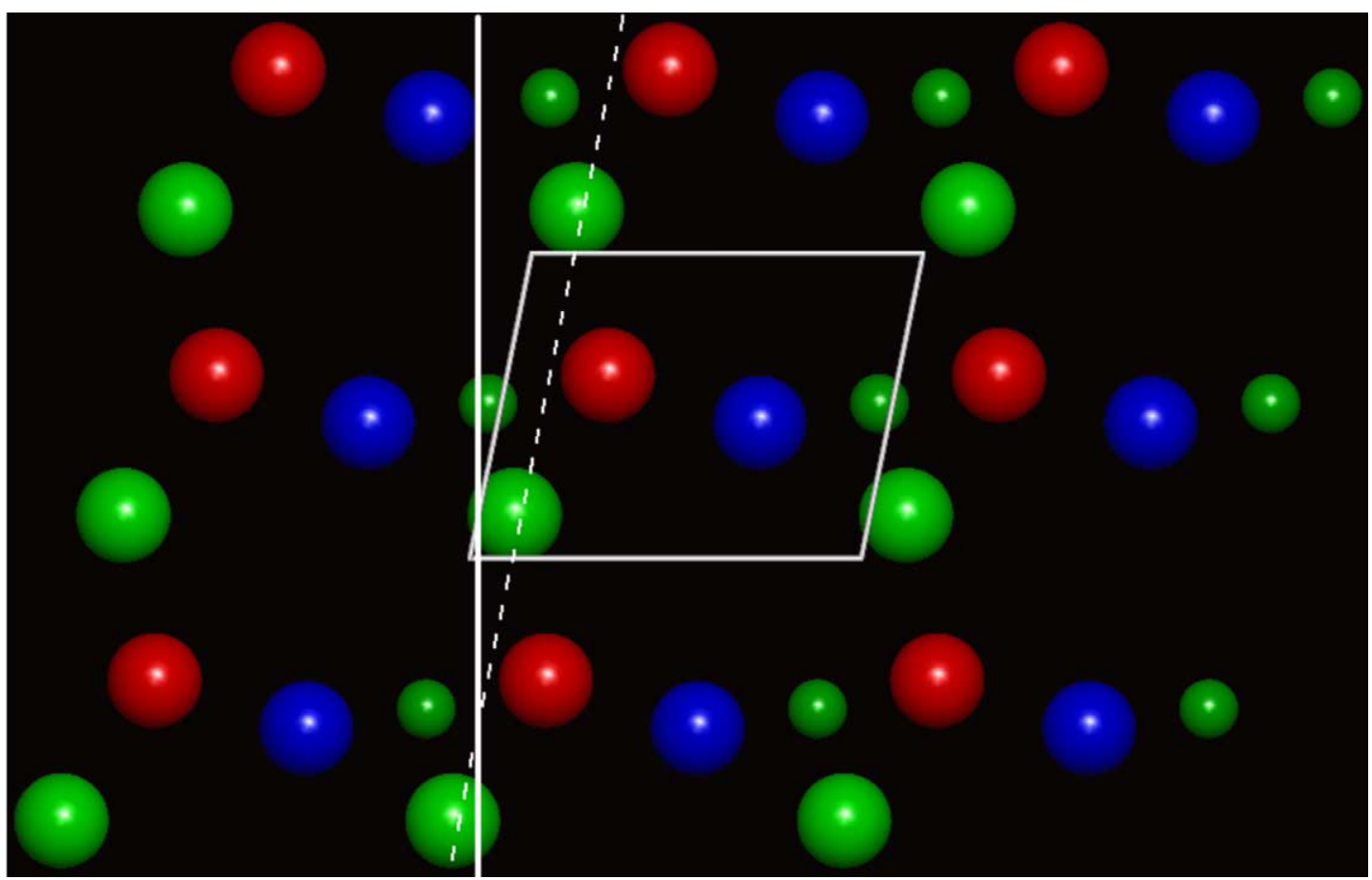

Fig. 4. (Continued).

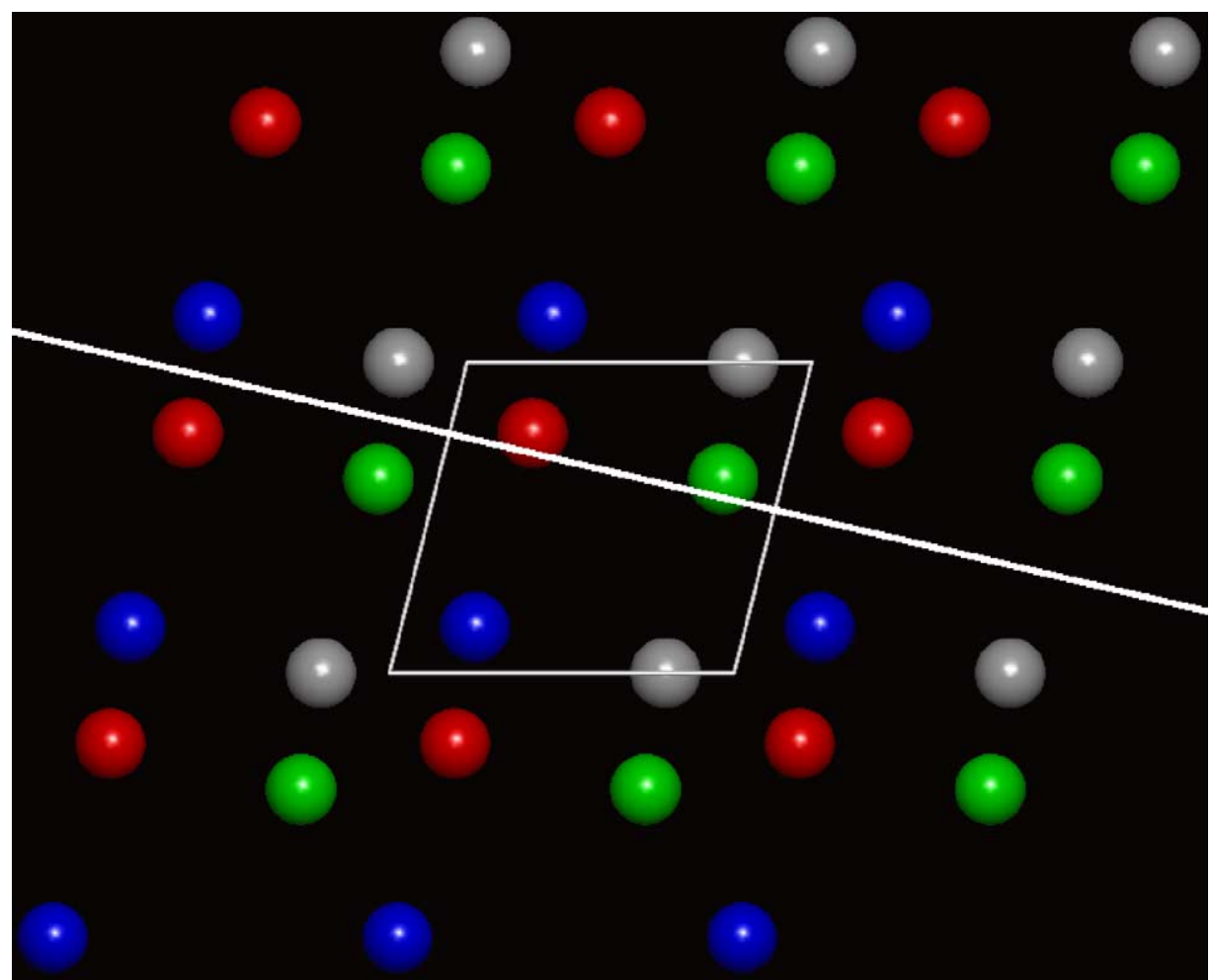

Fig. 5. The $\left(\begin{array}{lll}1 & 1 & 0\end{array}\right)$ face of the orthoclase. Minimum chiral indices $\left(I_{\mathrm{CA}}=0.52 \AA\right.$ and $\left.I_{\mathrm{CM}}=1.01 \AA\right)$ result from a mirror plane oriented perpendicular to $\left[\begin{array}{ll}1 & 0\end{array}\right]$.

measure of the intrinsic potential of crystalline surfaces to discriminate between chiral molecules. Surfaces with relatively large chiral indices have an inherently greater enantioselectve potential than those with indices near zero, and are perhaps worthy of special consideration in developing enantioselective chemical systems. However, no single chiral index is sufficient to characterize all interactions between a crystalline surface and a chiral molecule. Our two 


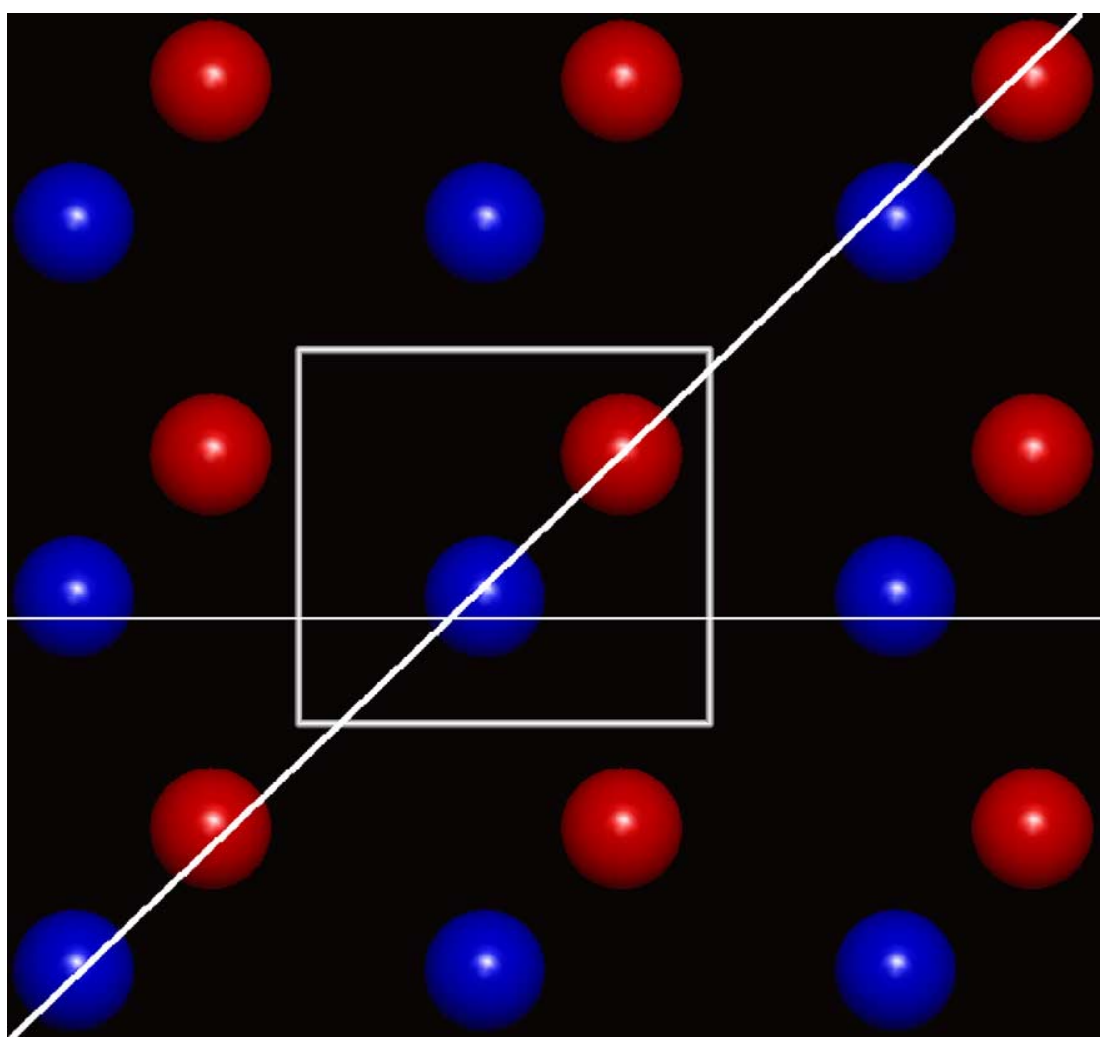

Fig. 6. The $\left(\begin{array}{lll}1 & 0 & 0\end{array}\right)$ surface of quartz has the largest chiral indices of the three common quartz crystal faces examined $\left(I_{\mathrm{CA}}=0.54 \AA\right.$ and $\left.I_{\mathrm{CM}}=0.59 \AA\right)$. Nevertheless, pseudo-mirror symmetry is evident for mirror planes oriented perpendicular to [1 0$]$ and [10 9].

proposed indices $I_{\mathrm{CA}}$ and $I_{\mathrm{CM}}$, for example, focus specifically on the deviations of a $3 \times 3$ array of surface unit cells from ideal mirror or glide plane symmetry. As such, these indices provide a measure of intermediate-range features, typically $15-45 \AA$ in length, but they may not adequately characterize interactions between these surfaces and chiral molecules at significantly larger or smaller scales.

In some instances, the scale of the calculation is not important. For example, if a 2D unit cell is orthogonal or if $a=b$, then the best-fit symmetry plane must be oriented parallel to a cell edge or a cell diagonal. In such a case $I_{\mathrm{CA}}$ and $I_{\mathrm{CM}}$ are independent of the number of surface unit cells considered.

In the case of a non-orthogonal unit cell, however, chiral indices may be a function of the number of unit cells included in the calculation. Consider, for example, a pseudo-orthogonal cell ( $a=3 \AA, b=5 \AA, \gamma=85^{\circ}$ ) with one atom at the origin, as illustrated in Fig. 7. In this case, the best-fit mirror is always oriented close to the pseudo-orthogonal cell edge, but the exact orientation of that mirror varies with the number of $2 \mathrm{D}$ unit cells under consideration, and the average and maximum deviations of atomic positions from that mirror increase as more unit cells are considered. Thus, in some cases $I_{\mathrm{CA}}$ and $I_{\mathrm{CM}}$ may vary depending on the scale of the calculation. Note that surfaces of this type may be more likely to interact selectively with large chiral molecules than small ones.
By contrast, a "kink site" on an FCC metal surface provides an effective chiral center at the scale of a few angstroms, even though much of the metal surface area may be intrinsically achiral. The local kinks, rather than the entire surface, provide chiral sites for enantioselectivity. Given these effects of scale, at least two alternative types of useful chiral indices could be devised with strategies similar to those outlined above.

\subsubsection{Three-point interactions}

Chiral selection of molecules requires three non-colinear points of interaction [34]. One could, therefore, define a chiral index based on the maximum enantiomeric mismatch of triangles formed by nearest-neighbor atoms. Such a chiral index thus probes short-range effects of chiral centers over a few Angstroms, rather than long-range effects over several unit cells.

The first step in calculating such a three-point chiral index is to identify all symmetrically distinct triangles formed by three non-colinear, nearest-neighbor surface atoms. Each atom in the surface unit cell will be incorporated into such triangles. For a surface unit cell with only one atom there exists only two different triangles, but this number increases significantly with additional atoms in the unit cell, especially if two or more different atomic species are present. For a surface to display a high degree of enantioselectivity two criteria must be met. 

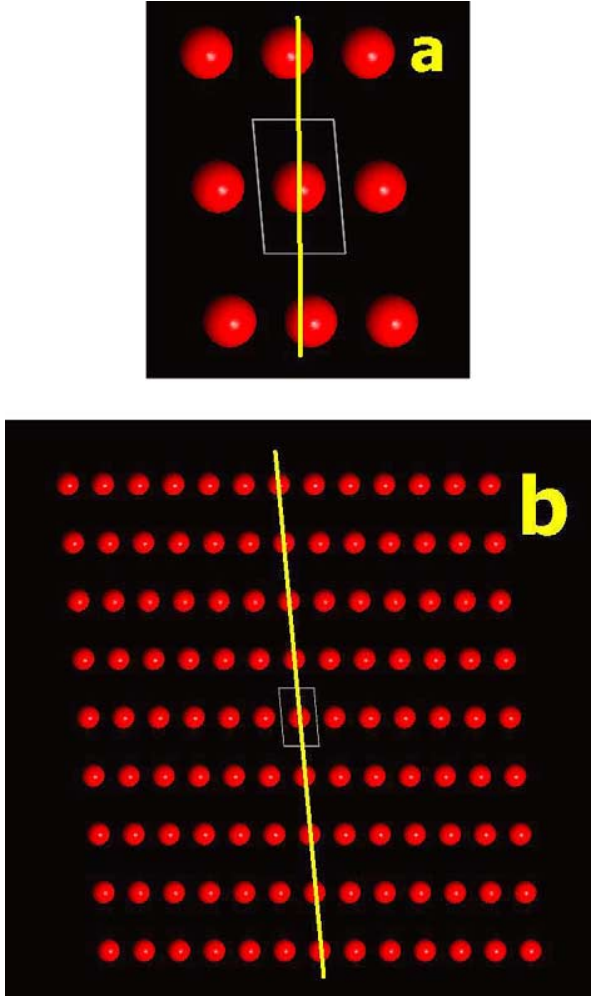

Fig. 7. A pseudo-orthogonal 2D surface unit cell (with mirror pseudo-symmetry) will have chiral indices, $I_{\mathrm{CA}}$ and $I_{\mathrm{CM}}$, that increase as more unit cells are included in the calculation. A $3 \times 3$ array of unit cells (a) has smaller chiral indices and a slightly different best-fit mirror orientation than a $13 \times 9$ array (b).

1. At least one triangular, three-atom configuration must deviate significantly from an isosceles triangle (which possesses mirror symmetry and thus is inherently achiral). Greater enantioselective potential will be associated with three-point configurations that deviate more from an isosceles triangle.

2. In addition, at least one of these non-isosceles, three-atom triangles must differ significantly from the mirror images of itself and of all other triangles. This restriction arises because a surface will be achiral if every non-isosceles triangle is present in both enantiomeric forms.

A three-point chiral index, therefore, will be based on the maximum misfit between a three-atom surface triangle and mirror images of itself and of all other surface triangles.

\subsubsection{Molecule-specific interactions}

Many studies of chiral selection are concerned with the efficient separation of specific molecular enantiomers. An alternative chiral index strategy, therefore, is to model the relative fit (or lack thereof) of a target chiral molecule on various crystalline surfaces versus that of its enantiomer. One could devise a misfit index that evaluates the conformity of any desired molecule (and that of its enantiomer) adsorbed onto various surfaces, based on likely three-point bonding configurations of the molecule and surface. The optimum surface for chiral resolution will have significantly different misfit parameters (one close to zero and the other large) for the two molecular enantiomers. This approach, which requires realistic atomic models of both the crystal surface and the chiral molecules, would facilitate the identification and engineering of surfaces for optimal selectivity.

\section{Conclusions}

Calculations of the chiral indices, $I_{\mathrm{CA}}$ and $I_{\mathrm{CM}}$, of various crystalline surfaces reveal several trends.

1. Achiral crystals often display strongly chiral surfaces. The (2 14) surface of calcite, the (1 10$)$ surface of diopside, and various high-index planes of FCC metals, for example, possess surfaces with no obvious mirror or glide plane symmetries, as indicated by their relatively large chiral indices. These surfaces thus are important targets for further study.

2. By contrast, the intrinsically chiral surfaces of quartz display relatively low chiral indices. Indeed, the ideal distribution of surface oxygen atoms on the (10 1) face is achiral.

3. In oxides and silicates, larger chiral indices are often associated with the presence of both terminal cations and anions. Thus, diopside (1 110$)$ faces have significantly greater chiral indices than quartz, which has only terminal oxygen atoms.

4. Relatively large chiral indices are often associated with stepped and kinked surfaces. This effect is demonstrated both by the high-index faces of FCC metals and by the (214) surfaces of calcite.

No one parameter can define the "degree of chirality" of a surface. Nevertheless, chiral indices provide a direct measure of the deviation of a surface from mirror or glide plane symmetries, and thus can prove useful in identifying promising surfaces for further study.

\section{Acknowledgements}

We gratefully acknowledge Aravind Asthagiri, Mary Ewell, Andrew Gellman, and David Sholl for invaluable discussions of this concept and constructive reviews of the manuscript. This work was supported by NSF grant EAR0229634, the NASA Astrobiology Institute and the Carnegie Institution of Washington.

\section{References}

[1] C.F. McFadden, P.S. Cremer, A.J. Gellman, Langmuir 12 (1996) 2483.

[2] D.S. Sholl, Langmuir 14 (1998) 862.

[3] K. Soai, et al., J. Am. Chem. Soc. 121 (1999) 11235. 
[4] A.J. Gellman, J.D. Horvath, M.T. Buelow, J. Mol. Catal. A 167 (2001) 3.

[5] G.A. Attard, J. Phys. Chem. B 105 (2001) 3158.

[6] B. Kahr, R.W. Gurney, Chem. Rev. 101 (2001) 893.

[7] M. Jacoby, Chem. Eng. News 80 (2002) 43.

[8] R.M. Hazen, D.S. Sholl, Nat. Mater. 2 (2003) 367.

[9] J.A. Switzer, et al., Nature 425 (2003) 490.

[10] N. Lahav, Biogenesis, Oxford, 1999.

[11] R.M. Hazen, G. Goodfriend, T. Filley, Proc. Natl. Acad. Sci. U.S.A. 98 (2001) 5487.

[12] P.S. Halasyamani, K.R. Poeppelmeier, Chem. Mater. 10 (1998) 2753.

[13] R.M. Hazen, in: G. Palyi, C. Zucchi, L. Caglioti (Eds.), Progress in Biological Chirality, Elsevier, New York, 2004, in press.

[14] K. Robinson, G.V. Gibbs, P.H. Ribbe, Science 172 (1971) 567.

[15] R.M. Hazen, L.W. Finger, Comparative Crystal Chemistry, Wiley, New York, 1982.

[16] R.M. Hazen, R.T. Downs, C.T. Prewitt, Rev. Mineral. Geochem. 41 (2000) 1.

[17] R.M. Thompson, R.T. Downs, Acta Cryst. B 57 (2001) 119.

[18] V.E. Heinrich, Prog. Surf. Sci. 14 (1983) 175.
[19] M.F. Hochella Jr., Rev. Mineral. 23 (1990) 87.

[20] U. Becker, M.F. Hochella Jr., E. Apra, Am. Mineral. 81 (1996) 1301.

[21] C.M. Koretsky, D.A. Sverjensky, N. Sahai, Am. J. Sci. 298 (1998) 349.

[22] A. Asthagiri, P.J. Feibelman, D.S. Sholl, Topics Catal. 18 (2002) 193.

[23] T.D. Power, A. Asthagiri, D.S. Sholl, Langmuir 18 (2002) 3737.

[24] D.L. Graf, Am. Mineral. 46 (1961) 1283.

[25] M. Cameron, S. Sueno, C.T. Prewitt, J.J. Papike, Am. Mineral. 58 (1973) 594.

[26] E. Prince, G. Donnay, R.F. Martin, Am. Mineral. 58 (1973) 500.

[27] K. Kihara, Eur. J. Mineral. 2 (1990) 63.

[28] R.T. Downs, M. Hall-Wallace, Am. Mineral. 88 (2003) 247.

[29] M.B. Boisen Jr., G.V. Gibbs, Mathematical Crystallography, revised edition, Mineralogical Society of America, Washington, 1990.

[30] R. Tsuchida, M. Kobayashi, A. Nakamura, J. Chem. Soc. Jpn. 56 (1935) 1339.

[31] W.A. Bonner, et al., Origins Life 6 (1975) 367.

[32] T.D. Powers, D.S. Sholl, J. Vac. Sci. Tech. A17 (1999) 1700.

[33] G.A. Attard, et al., J. Phys. Chem. B103 (1999) 1381.

[34] V.A. Davankov, Chirality 9 (1997) 99. 